\title{
THE NEUMANN LAPLACIAN OF A JELLY ROLL
}

\author{
BARRY SIMON
}

(Communicated by Paul S. Muhly)

\begin{abstract}
We consider the Laplacian with Neumann boundary conditions of a bounded connected region obtained by removing a suitable infinite spiral from an annulus. We show that the spectrum has an absolutely continuous component.
\end{abstract}

This note is a contribution to the study of the spectral properties of Neumann Laplacians, a subject of several recent papers [2-4]. Consider the curve, $\Gamma$, in $\mathbb{R}^{2}$ given in polar coordinates by

$$
r(\theta)=[3 \pi / 2+\operatorname{Arctan}(\theta)] / 2 \pi \quad-\infty<\theta<\infty,
$$

which is asymptotic to the circles $r=\frac{1}{2}$ (resp. $r=1$ ) as $\theta \rightarrow-\infty$ (resp. $\theta \rightarrow \infty)$.

Let $\Omega$ be the region

$$
\left\{(x, y) \in \mathbb{R}^{2} \mid \frac{1}{2}<r<1\right\} \backslash \Gamma,
$$

which is open, connected and bounded. Its boundary is $\Gamma \cup\left\{r=\frac{1}{2}\right\} \cup\{r=1\}$. Let $H=-\Delta_{N}^{\Omega}$ the Neumann Laplacian for $\Omega$. Since the circular parts of $\partial \Omega$ are singular points, we use the method of quadratic forms to define $H$. In fact, however, it could be defined by requiring classical $\partial \varphi / \partial n=0$ boundary conditions on (both sides of) $\Gamma$ and no boundary conditions on the circles because $\left\{\varphi \in D(H) \mid \operatorname{supp} \varphi \subset\{a<r<b\}\right.$ with $\left.\frac{1}{2}<a<b<1\right\}$ is a core for $H$.

Our main result here is

Theorem. (a) $\sigma(H)=[0, \infty)$;

(b) $\sigma_{\mathrm{ac}}(H)=[0, \infty)$ of uniform multiplicity 2 ;

(c) $\sigma_{\mathrm{sc}}(H)=\varnothing$;

(d) Any eigenvalue of $\mathrm{H}$ is of finite multiplicity and the only possible limit point of eigenvalues is $\infty$.

What is interesting is that $\Omega$ is a bounded region but $H$ still has absolutely continuous spectrum. It has been known, at least since the book of CourantHilbert [1], that even though Dirichlet Laplacians of bounded regions have

Received by the editors October 11, 1990.

1991 Mathematics Subject Classification. Primary 47A10, 35P99.

Research partially funded under NSF grant number DMS-8801918. 
purely discrete spectrum, there are bounded regions with $\sigma_{\text {ess }}\left(-\Delta_{N}^{\Omega}\right) \neq \varnothing$. But the Courant-Hilbert example has $\sigma_{\text {ess }}=\{0\}$ [3]. Recently Hempel, Seco, and Simon [3] constructed regions with $\sigma_{\text {ess }}\left(-\Delta_{N}^{\Omega}\right)=[0, \infty)$ but their examples have empty absolutely continuous spectrum.

In light of Davies-Simon [2] who discuss unbounded but finite volume regions whose $-\Delta_{N}^{\Omega}$ have absolutely continuous spectrum, our result here should not be surprising - in a real sense, our $\Omega$ here is just one of their regions "rolled up." That is why we think of $\Omega$ as a jelly roll, albeit one whose jelly, alas, is infinitely thin.

Proof of the theorem. We shift to polar coordinates $\theta, r$ with $\theta$ running from $-\infty$ to $\infty$. Explicitly, we let $\widetilde{\Omega}$ be $\left\{(\theta, r) \mid-\infty<\theta<\infty ; r_{-}(\theta)<r<r_{+}(\theta)\right\}$ with $r_{-}(\theta)=r(\theta)$ and $r_{+}(\theta)=r(\theta+2 \pi)$. There is an obvious one-toone map from $\Omega$ to $\widetilde{\Omega}$ under which $L^{2}\left(\Omega, d^{2} r\right)$ is unitarily equivalent to $L^{2}(\widetilde{\Omega}, r d r d \theta)$ and $H$ is equivalent to the quadratic form, $\widetilde{H}$, given by

$$
(g, \tilde{H} g)=\int\left(\left|\frac{\partial g}{\partial r}\right|^{2} r+\left|\frac{\partial g}{\partial \theta}\right|^{2} \frac{1}{r}\right) d r d \theta \text {. }
$$

As in [2], a special role is played by the functions $g(\theta, r)=g(\theta)$; then

$$
\|g\|^{2}=\int F(\theta)|g(\theta)|^{2} d \theta, \quad(g, \tilde{H} g)=\int G(\theta)\left|\frac{d g}{d \theta}(\theta)\right|^{2} d \theta,
$$

where $F(\theta)=\frac{1}{2}\left[r_{+}(\theta)^{2}-r_{-}(\theta)^{2}\right]$ and $G(\theta)=\ln \left[r_{+}(\theta) / r_{-}(\theta)\right]$. Since $r^{\prime}(\theta) \sim \theta^{-2}$ at infinity, $r_{+}(\theta)-r_{-}(\theta) \sim \theta^{-2}$ so, $F, G \sim \theta^{-2}$. Explicitly

$$
\begin{gathered}
r^{\prime}(\theta) \sim \pi^{-1}\left[\theta^{-2}-\theta^{-4}+O\left(|\theta|^{-6}\right)\right] ; \\
r(\theta)-r( \pm \infty) \sim \pi^{-1} \theta-\frac{1}{3} \pi^{-1} \theta^{-3}+O\left(|\theta|^{-5}\right) ; \\
F(\theta) \sim r( \pm \infty)\left[\frac{2}{\theta^{2}}+\frac{\beta}{\theta^{4}}+O\left(|\theta|^{-6}\right] ;\right. \\
G(\theta)=\frac{2}{\theta^{2}}+\frac{\alpha}{\theta^{4}}+O\left(|\theta|^{-6}\right) ; \\
G(\theta) / F(\theta)=r( \pm \infty)^{-1}\left[1+O\left(|\theta|^{-2}\right)\right] .
\end{gathered}
$$

In the usual way, $\widetilde{H}$ is unitarily equivalent to $\widehat{H}$ on $L^{2}(\mathbb{R}, d \theta)$ where

$$
\widetilde{H}=-\frac{1}{\sqrt{F}} \frac{d}{d \theta} \sqrt{F}\left(\frac{G}{F}\right) \sqrt{F} \frac{d}{d \theta} \frac{1}{\sqrt{F}}=-\frac{d}{d \theta}\left(\frac{G}{F}\right) \frac{d}{d \theta}+V(\theta),
$$

where $V(\theta) \sim \theta^{-2}$. Except for the $\theta$ dependence $G / F$ in $-d^{2} / d \theta^{2}$, the setup looks exactly like that in Davies-Simon [2]. Since (1) holds and the Enss theory easily accommodates principal part perturbations, our proof follows that in [2].

\section{REFERENCES}

1. R. Courant and D. Hilbert, Methods of mathematical physics, Interscience, New York, 1953.

2. E. B. Davies and B. Simon, Spectral properties of the Neumann Laplacian of horns, preprint. 
3. R. Hempel, L. A. Seco, and B. Simon, The essential spectrum of Neumann Laplacians on some bounded singular domains, preprint.

4. V. Jaksic, S. Molchhonov, and B. Simon, Eigenvalue asymptotics of the Neumann Laplacian of the manifolds and regions with cusps, in preparation.

Division of Physics, Mathematics, and Astronomy, California Institute of Technology, 253-37, Pasadena, California 91125 